\title{
INVERSE CURVATURE FLOW IN ANTI-DE SITTER-SCHWARZSCHILD MANIFOLD
}

\author{
SIYUAN LU
}

\begin{abstract}
In this paper, we consider the inverse hessian quotient curvature flow with star-shaped initial hypersurface in anti-de Sitter-Schwarzschild manifold. We prove that the solution exists for all time, and the second fundamental form converges to identity exponentially fast.
\end{abstract}

\section{INTRODUCTION}

Curvature flows of compact hypersurfaces in Riemannian manifolds have been extensively studied in the last 30 years. In the case of Euclidean space, for contracting flow, Huisken [13] considered

$$
\dot{X}=-H \nu
$$

where $H$ is the mean curvature. He proved that the solution exists for all time and the normalized flow converges to a round sphere if the initial hypersurface is convex.

This result is later generalized by Andrews [1] for a large class of curvature flow. More specificly, Andrews considered

$$
\dot{X}=-F \nu
$$

where $F$ is a concave function of homogeneous degree one, evaluated at the principal curvature.

For expanding flow, Gerhardt [7] and Urbas [20] considered

$$
\dot{X}=\frac{\nu}{F}
$$

where $F$ is a concave function of homogeneous degree one, evaluated at the principal curvature. They proved that the solution exists for all time and the normalized flow converges to a round sphere if the initial hypersurface is star-shaped and lies in a certain convex cone.

A natural question is whether these results remain true if the ambient space is no longer Euclidean space. For contraction flow (1.1) and (1.2), Huisken [14] and Andrews [2] generalized their results to certain ambient space respectively.

The case of expanding flow (1.3) is in fact more subtle as the assumption on initial hypersurface is weaker. In the case of space form, Gerhardt [8, 9] proved the solution exists for all time and the second fundamental form converges in hyperbolic space and sphere space, see also earlier work by Ding [6]. More recently, Brendle-Hung-Wang [3] and Scheuer [19] proved that the same results hold in anti-de Sitter-Schwarzschild manifold and a class of warped product manifold for inverse mean curvature flow, which is

$$
\dot{X}=\frac{\nu}{H}
$$

Research of the author was supported in part by CSC fellowship and Schulich Graduate fellowship. 
However, as pointed out by Neves [17] and Hung-Wang in [15], for inverse mean curvature flow, the rescaled hypersurface is not necessary a round sphere in anti-de SitterSchwarzschild manifold and in hyperbolic space.

Inverse curvature flows can be used to prove various inequalities. Guan-Li [10] generalized Alexandrov-Fenchel inequalities for star-shaped $k$-convex hypersurface in Euclidean space using inverse curvature flow (1.3) in Euclidean space. Recently, Brendle-Hung-Wang [3] generalized Alexandrov-Fenchel inequality for $k=1$ (which they call Minkowski inequality) in anti-de Sitter-Schwarzschild manifold by inverse mean curvature flow (1.4). The inequality is further used to prove a Penrose inequality in General Relativity in [4]. More recently, Li-Wei-Xiong [16] and Ge-Wang-Wu[12] generalized the hyperbolic Alexandrov-Fenchel inequality using inverse curvature flow (1.3) in hyperbolic space.

Motivated by the results above, we consider inverse curvature flow in anti-de SitterSchwarzschild manifold. The anti-de Sitter-Schwarzschild manifold is a manifold $N=$ $\mathbb{S}^{n} \times\left[s_{0}, \infty\right)$ equipped with the following Riemannian metric

$$
\bar{g}=\frac{1}{1-m s^{1-n}+s^{2}} d s^{2}+s^{2} g_{\mathbb{S} n}
$$

where $s_{0}$ is the unique positive solution of the equation $1-m s^{1-n}+s^{2}=0$. By a change of variable, we have

$$
\bar{g}=d r^{2}+\phi^{2}(r) g_{\mathbb{S}^{n}}
$$

where $\phi$ satisfies $\phi^{\prime}=\sqrt{1-m \phi^{1-n}+\phi^{2}}$.

The anti-de Sitter-Schwarzschild manifold is thus a special case of warped product manifold. Moreover, the sectional curvature of $(N, \bar{g})$ approach -1 near infinity exponentially fast and the scalar curvature is of constant $-n(n+1)$. This feature will play an essential role in the proof of our theorem.

To state our theorem, we need the following definition of Garding's $\Gamma_{k}$ cone $\Gamma_{k}=\left\{\left(\kappa_{i}\right) \in\right.$ $\left.\mathbb{R}^{n} \mid \sigma_{j}>0,0 \leq j \leq k\right\}$, where $\sigma_{j}$ is the $j$-th elementrary symmetric function. We say a hypersurface is $k$-convex if the principal curvature $\left(\kappa_{i}\right) \in \Gamma_{k}$.

We now state our main theorem:

Theorem 1.1. Let $\Sigma_{0}^{n}$ be a star-shaped, $k$-convex closed hypersurface in $N^{n+1}$, where $N^{n+1}$ is an anti-de Sitter-Schwarzschild manifold, consider the evolution equation

$$
\dot{X}=\frac{\nu}{F}
$$

where $\nu$ is the ourward unit normal and $F=n \frac{C_{n}^{k-1}}{C_{n}^{k}} \frac{\sigma_{k}}{\sigma_{k-1}}$ which is evaluated at the principal curvature of $\Sigma_{t}$. Then the solution exists for all time $t$, and the second fundamental form satisfies

$$
\left|h_{j}^{i}-\delta_{j}^{i}\right| \leq C e^{-\frac{2}{n} t}
$$

where $C$ depends on the $\Sigma_{0}, n, k$.

The organization of the paper is as follows: in section 2, we give some preliminaries about warped product space and anti-de Sitter-Schwarzschild manifold, we also prove the $C^{0}$ estimate. In section 3 , we derive the evolution equations and give the $C^{1}$ estimate. In section 4 and 5 , we estimate the bound for $F$ and the principal curvature respectively. In section 6 , we prove that the second fundamental form converges to identity. 
After submitting the paper, we have learned that Chen-Mao [5] independently proved the main theorem above.

\section{Preliminaries}

In this section, we give some basic properties of hypersurface in warped product space. Let $N^{n+1}$ be a warped product space, with the metric

$$
g^{N}:=d s^{2}=d r^{2}+\phi^{2}(r) \sigma_{i j}
$$

where $\sigma_{i j}$ is the standard metric of $\mathbb{S}^{n}$.

Define

$$
\Phi(r)=\int_{0}^{r} \phi(\rho) d \rho, \quad V=\phi(r) \frac{\partial}{\partial r}
$$

We state some well-known lemmas, see [11] with some modification.

Lemma 2.1. The vector field $V$ satisfies $D_{i} V_{j}=\phi^{\prime}(r) g_{i j}^{N}$, where $D$ is the covariant derivative with respect to the metric $g^{N}$.

Lemma 2.2. Let $M^{n} \subset N^{n+1}$ be a closed hypersurface with induced metric $g$, then $\left.\Phi\right|_{M}$ satisfies,

$$
\nabla_{i} \nabla_{j} \Phi=\phi^{\prime}(r) g_{i j}-h_{i j}\langle V, \nu\rangle,
$$

where $\nabla$ is the covariant derivative with respect to $g, \nu$ is the outward unit normal and $h_{i j}$ is the second fundamental form of the hypersurface.

We now state the Gauss equation Codazzi equation,

$$
\begin{gathered}
R_{i j k l}=\bar{R}_{i j k l}+\left(h_{i k} h_{j l}-h_{i l} h_{j k}\right) \\
\nabla_{k} h_{i j}-\nabla_{j} h_{i k}=\bar{R}_{\nu i j k}
\end{gathered}
$$

and the interchanging formula

$$
\begin{aligned}
\nabla_{i} \nabla_{j} h_{k l}= & \nabla_{k} \nabla_{l} h_{i j}-h_{l}^{m}\left(h_{i m} h_{k j}-h_{i j} h_{m k}\right)-h_{j}^{m}\left(h_{m i} h_{k l}-h_{i l} h_{m k}\right) \\
& +h_{l}^{m} \bar{R}_{i k j m}+h_{j}^{m} \bar{R}_{i k l m}+\nabla_{k} \bar{R}_{i j l \nu}+\nabla_{i} \bar{R}_{j k l \nu}
\end{aligned}
$$

Define the support function $u=\langle V, \nu\rangle$, and we have

\section{Lemma 2.3.}

$$
\begin{aligned}
\nabla_{i} u & =g^{k l} h_{i k} \nabla_{l} \Phi, \\
\nabla_{i} \nabla_{j} u & =g^{k l} \nabla_{k} h_{i j} \nabla_{l} \Phi+\phi^{\prime} h_{i j}-\left(h^{2}\right)_{i j} u+g^{k l} \nabla_{l} \Phi \bar{R}_{\nu j k i},
\end{aligned}
$$

where $\left(h^{2}\right)_{i j}=g^{k l} h_{i k} h_{j l}, \bar{R}_{\nu j k i}$ is the curvature of ambient space.

Proof. We only need to prove the equality at one point, thus we have $g_{i j}=\delta_{i j}$ and $\nabla_{i} u=$ $D_{i}\langle V, \nu\rangle=\left\langle D \Phi, D_{i} \nu\right\rangle=h_{i k} D_{k} \Phi$.

$$
\begin{aligned}
\nabla_{i} \nabla_{j} u & =\nabla_{i} h_{j k} \nabla_{k} \Phi+h_{j k} \nabla_{i} \nabla_{k} \Phi \\
& =\nabla_{i} h_{j k} \nabla_{k} \Phi+h_{j k}\left(\phi^{\prime} g_{i k}-h_{i k} u\right) \\
& =\left(\nabla_{k} h_{i j}+\bar{R}_{\nu j k i}\right) \nabla_{k} \Phi+\phi^{\prime} h_{i j}-\left(h^{2}\right)_{i j} u,
\end{aligned}
$$

where Codazzi equation (2.3) is used in the last equality, thus by the tensorial property, we have the lemma. 
As to the curvature, we have the following curvature estimates, for proof, we refer readers to $[3]$.

Lemma 2.4. The sectional curvature satisfies

$$
\begin{aligned}
& \bar{R}\left(\partial_{i}, \partial_{j}, \partial_{k}, \partial_{l}\right)=\phi^{2}\left(1-\phi^{\prime 2}\right)\left(\sigma_{i k} \sigma_{j l}-\sigma_{i l} \sigma_{j k}\right) \\
& \bar{R}\left(\partial_{i}, \partial_{r}, \partial_{j}, \partial_{r}\right)=-\phi \phi^{\prime \prime} \sigma_{i j}
\end{aligned}
$$

where $\partial_{i}$ is the standard frame on $\mathbb{S}^{n}$ and $\sigma_{i j}$ is the standard metric of $\mathbb{S}^{n}$.

Now, back to our case that $N$ is an anti-de Sitter-Schwarzschild manifold,

Lemma 2.5. Let $N$ be an anti-de Sitter-Schwarzschild manifold, we have

$$
\phi(r)=\sinh (r)+\frac{m}{2(n+1)} \sinh ^{-n}(r)+O\left(\sinh ^{-n-2}(r)\right)
$$

and

$$
\begin{gathered}
\bar{R}_{\alpha \beta \gamma \mu}=-\delta_{\alpha \gamma} \delta_{\beta \mu}+\delta_{\alpha \mu} \delta_{\beta \gamma}+O\left(e^{-(n+1) r}\right) \\
\bar{\nabla}_{\rho} \bar{R}_{\alpha \beta \gamma \mu}=O\left(e^{-(n+1) r}\right)
\end{gathered}
$$

where $\left\{e_{\alpha}\right\}$ is an orthonormal frame in $N$.

We also need the following two lemmas regarding to $\sigma_{k}$. These two lemmas are well known, for completeness, we add the proof here.

Lemma 2.6. let $F=n \frac{C_{n}^{k-1}}{C_{n}^{k}} \frac{\sigma_{k}}{\sigma_{k-1}}$, thus $F$ is of homogeneous degree 1 , and $F(I)=n$, then we have

$$
\sum_{i} F^{i i} \lambda_{i}^{2} \geq \frac{F^{2}}{n}
$$

Proof. We first consider the term $\sigma_{l}^{i i} \lambda_{i}^{2}$, we have

$$
\sigma_{l}^{i i} \lambda_{i}^{2}=\sigma_{1} \sigma_{l}-(l+1) \sigma_{l+1}
$$

Let $G=\frac{\sigma_{k}}{\sigma_{k-1}}$, by (2.6) and Newton-Mclaraun ineqaulity, we have

$$
\begin{aligned}
\sum_{i} G^{i i} \lambda_{i}^{2} & =\sum_{i}\left(\frac{\sigma_{k}^{i i}}{\sigma_{k-1}}-\frac{\sigma_{k} \sigma_{k-1}^{i i}}{\sigma_{k-1}^{2}}\right) \lambda_{i}^{2} \\
& =\frac{\sigma_{1} \sigma_{k}-(k+1) \sigma_{k+1}}{\sigma_{k-1}}-\frac{\sigma_{k}\left(\sigma_{1} \sigma_{k-1}-k \sigma_{k}\right)}{\sigma_{k-1}^{2}} \\
& =\frac{k \sigma_{k}^{2}-(k+1) \sigma_{k-1} \sigma_{k+1}}{\sigma_{k-1}^{2}} \\
& \geq \frac{k \sigma_{k}^{2}}{(n-k+1) \sigma_{k-1}^{2}} \\
& =\frac{C_{n}^{k-1}}{C_{n}^{k}}\left(\frac{\sigma_{k}}{\sigma_{k-1}}\right)^{2}
\end{aligned}
$$


thus

$$
\sum_{i} F^{i i} \lambda_{i}^{2} \geq n\left(\frac{C_{n}^{k-1}}{C_{n}^{k}}\right)^{2}\left(\frac{\sigma_{k}}{\sigma_{k-1}}\right)^{2}=\frac{F^{2}}{n}
$$

Lemma 2.7. Let $F=n \frac{C_{n}^{k-1}}{C_{n}^{k}} \frac{\sigma_{k}}{\sigma_{k-1}}$ and $\left(\lambda_{i}\right) \in \Gamma_{k}$, then

$$
n \leq \sum_{i} F^{i i} \leq n k
$$

Proof. Let $G=\frac{\sigma_{k}}{\sigma_{k-1}}$, we have

$$
\begin{aligned}
\sum_{i} G^{i i} & =\sum_{i}\left(\frac{\sigma_{k}^{i i}}{\sigma_{k-1}}-\frac{\sigma_{k} \sigma_{k-1}^{i i}}{\sigma_{k-1}^{2}}\right) \\
& =(n-k+1)-(n-k+2) \frac{\sigma_{k} \sigma_{k-2}}{\sigma_{k-1}^{2}} \\
& \geq \frac{n-k+1}{k}
\end{aligned}
$$

by Newton-Mclaraun inequality.

For the second inequality,

$$
\begin{aligned}
\sum_{i} G^{i i} & =\sum_{i}\left(\frac{\sigma_{k}^{i i}}{\sigma_{k-1}}-\frac{\sigma_{k} \sigma_{k-1}^{i i}}{\sigma_{k-1}^{2}}\right) \\
& =(n-k+1)-(n-k+2) \frac{\sigma_{k} \sigma_{k-2}}{\sigma_{k-1}^{2}} \\
& \leq n-k+1
\end{aligned}
$$

as $\left(\lambda_{i}\right) \in \Gamma_{k}$. The lemma then follows.

Since the initial hypersurface is star-shaped, we can consider it as a graph on $\mathbb{S}^{n}$, i.e. $X=(x, r)$ where $x$ is the coordinate on $\mathbb{S}^{n}, r$ is the radius, by taking derivatives, we have

$$
\begin{aligned}
X_{i} & =\partial_{i}+r_{i} \partial_{r} \\
g_{i j} & =r_{i} r_{j}+\phi^{2} \sigma_{i j}
\end{aligned}
$$

and

$$
\nu=\frac{1}{v}\left(-\frac{r^{i}}{\phi^{2}} \partial_{i}+\partial_{r}\right)
$$

where $\nu$ is the unit normal vector, $v=\left(1+\frac{|\nabla r|^{2}}{\phi^{2}}\right)^{\frac{1}{2}}$, note that all the derivatives are on $\mathbb{S}^{n}$.

Thus

$$
\frac{d r}{d t}=\frac{1}{F v}, \dot{x}^{i}=-\frac{r^{i}}{\phi^{2} F v}
$$

we have

$$
\frac{\partial r}{\partial t}=\frac{d r}{d t}-r_{j} \dot{x}^{j}=\frac{v}{F}
$$


By a direct computation, c.f. (2.6) in [6] we have

$$
h_{i j}=\frac{1}{v}\left(-r_{i j}+\phi \phi^{\prime} \sigma_{i j}+\frac{2 \phi^{\prime} r_{i} r_{j}}{\phi}\right)
$$

Now we consider a function

$$
\varphi=\int_{r_{0}}^{r} \frac{1}{\phi}
$$

thus

$$
\varphi_{i}=\frac{r_{i}}{\phi}, \varphi_{i j}=\frac{r_{i j}}{\phi}-\frac{\phi^{\prime} r_{i} r_{j}}{\phi^{2}}
$$

If we write everything in terms of $\varphi$, we have

$$
\frac{\partial \varphi}{\partial t}=\frac{v}{\phi F}
$$

and

$$
v=\left(1+|D \varphi|^{2}\right)^{\frac{1}{2}}, g_{i j}=\phi^{2}\left(\varphi_{i} \varphi_{j}+\sigma_{i j}\right), g^{i j}=\phi^{-2}\left(\sigma^{i j}-\frac{\varphi^{i} \varphi^{j}}{v^{2}}\right) .
$$

Moreover,

$$
\begin{aligned}
h_{i j} & =\frac{\phi}{v}\left(\phi^{\prime}\left(\sigma_{i j}+\varphi_{i} \varphi_{j}\right)-\varphi_{i j}\right), \\
h_{j}^{i} & =g^{i k} h_{k j}=\frac{\phi^{\prime}}{\phi v} \delta_{j}^{i}-\frac{1}{\phi v} \tilde{\sigma}^{i k} \varphi_{k j}
\end{aligned}
$$

where $\tilde{\sigma}^{i j}=\sigma^{i j}-\frac{\varphi^{i} \varphi^{j}}{v^{2}}$.

We now give the $C^{0}$ estimate.

Lemma 2.8. Let $\bar{r}(t)=\sup _{\mathbb{S}^{n}} r(\cdot, t)$ and $\underline{r}(t)=\inf _{\mathbb{S}^{n}} r(\cdot, t)$, then we have

$$
\begin{aligned}
& \phi(\bar{r}(t)) \leq e^{t / n} \phi(\bar{r}(0)) \\
& \phi(\underline{r}(t)) \geq e^{t / n} \phi(\underline{r}(0))
\end{aligned}
$$

Proof. Recall that $\frac{\partial r}{\partial t}=\frac{v}{F}$, where $F$ is a normalized operator on $\left(h_{j}^{i}\right)$. At the point where the function $r(\cdot, t)$ attains its maximum, we have $\nabla r=0,\left(r_{i j}\right) \leq 0$, from (2.12), we deduce that $\nabla \varphi=0,\left(\varphi_{i j}\right) \leq 0$ at the maximum point. From (2.15), we have $\left(h_{j}^{i}\right) \geq\left(\frac{\phi^{\prime}}{\phi} \delta_{j}^{i}\right)$, where we may assume $\left(g_{i j}\right)$ and $\left(h_{i j}\right)$ is diagonalized if necessary. Since $F$ is homogeneous of degree 1 , and $F(1, \cdots, 1)=n$, we have

$$
v^{2}=1+|\nabla \varphi|^{2}=1, F\left(h_{j}^{i}\right) \geq \frac{\phi^{\prime}}{\phi} F\left(\delta_{j}^{i}\right)=\frac{n \phi^{\prime}}{\phi},
$$

thus

$$
\frac{d}{d t} \bar{r}(t) \leq \frac{\phi(\bar{r}(t))}{n \phi^{\prime}(\bar{r}(t))}
$$

i.e.

$$
\frac{d}{d t} \log \phi(\bar{r}(t)) \leq \frac{1}{n}
$$

which yields to the first inequality. Similarly, we can prove the second inequality, thus we have the lemma. 


\section{Evolution equations And $C^{1}$ estimate}

Before we go on with the estimate, let's derive some evolution equations first.

$$
\begin{gathered}
\dot{g}_{i j}=\frac{2 h_{i j}}{F}, \quad \dot{\nu}=\frac{g^{i j} F_{i} e_{j}}{F^{2}} \\
\dot{h}_{j}^{i}=-\frac{1}{F} h_{k}^{i} h_{j}^{k}-\nabla^{i} \nabla_{j}\left(\frac{1}{F}\right)-\frac{1}{F} \bar{R}_{\nu j \nu}^{i}
\end{gathered}
$$

Together with the interchanging formula (2.4), we have

$$
\begin{aligned}
\dot{h}_{j}^{i} & =-\frac{1}{F} h_{k}^{i} h_{j}^{k}+\frac{F^{p q, r s} h_{p q}{ }^{i} h_{r s j}}{F^{2}}-\frac{2 F^{p q} h_{p q}{ }^{i} F^{r s} h_{r s j}}{F^{3}}-\frac{1}{F} \bar{R}_{\nu j \nu}^{i} \\
& +\frac{g^{k i} F^{p q}}{F^{2}}\left(h_{k j, p q}-h_{q}^{m}\left(h_{k m} h_{p j}-h_{k j} h_{m p}\right)-h_{j}^{m}\left(h_{m k} h_{p q}-h_{k q} h_{m p}\right)\right. \\
& \left.+h_{q}^{m} \bar{R}_{k p j m}+h_{j}^{m} \bar{R}_{k p q m}+\nabla_{p} \bar{R}_{k j q \nu}+\nabla_{k} \bar{R}_{j p q \nu}\right)
\end{aligned}
$$

where $F^{i j}=\frac{\partial F}{\partial h_{p q}}$ and $F^{p q, r s}=\frac{\partial^{2} F}{\partial h_{p q} \partial h_{r s}}$.

For later purpose, we consider the function $u=\left\langle\phi \partial_{r}, \nu\right\rangle=\frac{\phi}{v}$, which can be seen as the support function. We derive the following equation.

$$
\dot{u}=\frac{\phi^{\prime}}{F}+\frac{\phi g^{i j} F_{i} r_{j}}{F^{2}}
$$

Now, we need to consider the curvature term. By Lemma 2.4, (2.7) and (2.8), we have

$$
\begin{gathered}
\bar{R}_{k \nu j \nu}=\left(\frac{1}{v^{2}} \delta_{k j}+\frac{2 r_{k} r_{j}}{\phi^{2} v^{2}}+\frac{r_{k} r_{j}|\nabla r|^{2}}{\phi^{4} v^{2}}\right)\left(-\phi \phi^{\prime \prime}\right)+\frac{\left(|\nabla r|^{2} \delta_{k j}-r_{k} r_{j}\right)}{\phi^{2} v^{2}}\left(1-\phi^{\prime 2}\right) \\
\bar{R}_{\nu j n k}=\frac{r_{n} \delta_{j k}}{v}\left(-\phi \phi^{\prime \prime}-\left(1-\left(\phi^{\prime}\right)^{2}\right)\right)+\frac{r_{k} \delta_{j n}}{v}\left(\phi \phi^{\prime \prime}+\left(1-\phi^{\prime 2}\right)\right)
\end{gathered}
$$

Note that $g^{m n}=\phi^{-2}\left(\sigma_{m n}-\frac{r^{m} r^{n}}{v^{2} \phi^{2}}\right)$, thus

$$
g^{m n} \nabla_{m} \Phi \bar{R}_{\nu j n k}=\left(\frac{|\nabla r|^{2} \delta_{j k}-r_{j} r_{k}}{\phi v^{3}}\right)\left(-\phi \phi^{\prime \prime}-\left(1-\phi^{\prime 2}\right)\right)
$$

Lemma 3.1. Along the flow, $|\dot{\varphi}| \leq C$, where $C$ depends on $\Sigma_{0}, n, k$.

Proof. By (2.13) and (2.15), we have

$$
\frac{\partial \varphi}{\partial t}=\frac{v^{2}}{F\left(\phi^{\prime} \delta_{i j}-\tilde{\sigma}^{i k} \varphi_{k j}\right)}=\frac{1}{G}
$$

Let $G^{i j}=\frac{\partial G}{\partial \varphi_{i j}}, G^{k}=\frac{\partial G}{\partial \varphi_{k}}$, then

$$
G^{i j}=-\frac{1}{v^{2}} F_{l}^{i} \tilde{\sigma}^{l j}
$$

thus

$$
\frac{\partial \dot{\varphi}}{\partial t}=-\frac{\dot{G}}{G^{2}}=\frac{1}{v^{2} G^{2}}\left(F_{l}^{i} \tilde{\sigma}^{l j} \dot{\varphi}_{i j}-v^{2} G^{k} \dot{\varphi}_{k}-F_{i}^{i} \phi \phi^{\prime \prime} \dot{\varphi}\right)
$$

By maximum principle, we conclude that $|\dot{\varphi}|$ is bounded above. 
Lemma 3.2. Along the flow, $|\nabla \varphi| \leq C$, where $C$ depends on $\Sigma_{0}, n, k$. In addition, if $F$ is bounded above, we have $|\nabla \varphi| \leq C e^{-\alpha t}$, where $\alpha$ depends on $\sup F$ and $n$.

Proof. By (2.13) and (2.15), we have

$$
\frac{\partial \varphi}{\partial t}=\frac{v^{2}}{F\left(\phi^{\prime} \delta_{i j}-\tilde{\sigma}^{i k} \varphi_{k j}\right)}=\frac{1}{G}
$$

Let $G^{i j}=\frac{\partial G}{\partial \varphi_{i j}}, G^{k}=\frac{\partial G}{\partial \varphi_{k}}$, then

$$
G^{i j}=-\frac{1}{v^{2}} F_{l}^{i} \tilde{\sigma}^{l j}
$$

Let $\omega=\frac{1}{2}|\nabla \varphi|^{2}$, we have

$$
\frac{\partial \omega}{\partial t}=-\frac{\varphi^{k}}{G^{2}} \nabla_{k} G=\frac{1}{v^{2} G^{2}}\left(F_{l}^{i} \tilde{\sigma}^{l j} \varphi^{k} \varphi_{i j k}-v^{2} G^{k} \omega_{k}-2 F_{i}^{i} \phi \phi^{\prime \prime} \omega\right)
$$

We want to write the term $\tilde{\sigma}^{l j} \varphi_{i j k}$ in terms of second derivative of $\omega$. Note that

$$
\begin{aligned}
\omega_{i j} & =\varphi_{k i j} \varphi^{k}+\varphi_{k i} \varphi_{j}^{k} \\
& =\varphi_{i j k} \varphi^{k}+\left(\sigma_{i j} \sigma_{k p}-\sigma_{i k} \sigma_{j p}\right) \varphi^{p} \varphi^{k}+\varphi_{k i} \varphi_{j}^{k} \\
& =\varphi_{i j k} \varphi^{k}+\sigma_{i j}|\nabla \varphi|^{2}-\varphi_{i} \varphi_{j}+\varphi_{k i} \varphi_{j}^{k}
\end{aligned}
$$

and

$$
\tilde{\sigma}^{l j}\left(\sigma_{i j}|\nabla \varphi|^{2}-\varphi_{i} \varphi_{j}\right)=\delta_{i}^{l}|\nabla \varphi|^{2}-\varphi_{i} \varphi^{l}
$$

Thus we have

$$
\frac{\partial w}{\partial t}=\frac{1}{v^{2} G^{2}}\left(F_{l}^{i} \tilde{\sigma}^{l j} \omega_{i j}-F_{i}^{i}|\nabla \varphi|^{2}+F_{l}^{i} \varphi_{i} \varphi^{l}-v^{2} G^{k} \omega_{k}-2 F_{i}^{i} \phi \phi^{\prime \prime} \omega\right)-\frac{1}{v^{2} G^{2}} F_{l}^{i} \tilde{\sigma}^{l j} \varphi_{k i} \varphi_{j}^{k}
$$

Note that $-F_{i}^{i}|\nabla \varphi|^{2}+F_{l}^{i} \varphi_{i} \varphi^{l} \leq 0$ and $-F_{l}^{i} \tilde{\sigma}^{l j} \varphi_{k i} \varphi_{j}^{k} \leq 0$, thus by the maximum principle, we have

$$
\omega(\cdot, t) \leq \sup \omega_{0}
$$

More pricisely, if $F \leq C$, consider the test function $\tilde{\omega}=\omega e^{\lambda t}$, thus at the maximum point of $\tilde{\omega}$, we have

$$
\begin{aligned}
0 & \leq \frac{\partial \omega}{\partial t} e^{\lambda t}+\lambda \omega e^{\lambda t} \leq \omega e^{\lambda t}\left(\frac{-2 F_{i}^{i} \phi \phi^{\prime \prime}}{v^{2} G^{2}}+\lambda\right) \\
& =\omega e^{\lambda t}\left(\frac{-2 F_{i}^{i}\left(h_{j}^{i}\right) \phi^{\prime \prime}}{\phi F^{2}\left(h_{j}^{i}\right)}+\lambda\right) \\
& \leq \omega e^{\lambda t}\left(\frac{-2 n \phi^{\prime \prime}}{\phi F^{2}\left(h_{j}^{i}\right)}+\lambda\right) \leq 0
\end{aligned}
$$

if $0<\lambda \leq \frac{2 n}{\sup ^{2} F} \leq \frac{2 n \phi^{\prime \prime}}{\phi \sup ^{2} F}$, we have used Lemma 2.7 in last line. By maximum principle,

$$
|\nabla \varphi| \leq C e^{-\alpha t}
$$

where $0<\alpha \leq \frac{n}{\sup ^{2} F}$. 


\section{BOUND FOR $F$}

Lemma 4.1. Along the flow, $F \leq C$, where $C$ depends on $\Sigma_{0}, n, k$.

Proof. By (3.2), we have

$$
\begin{aligned}
\dot{F} & =F_{i}^{j}\left(-\frac{1}{F} h_{k}^{i} h_{j}^{k}-\nabla^{i} \nabla_{j}\left(\frac{1}{F}\right)-\frac{1}{F} \bar{R}_{\nu j \nu}^{i}\right) \\
& =F_{i}^{j}\left(-\frac{1}{F} h_{k}^{i} h_{j}^{k}+\frac{\nabla^{i} \nabla_{j} F}{F^{2}}-2 \frac{\nabla^{i} F \nabla_{j} F}{F^{3}}-\frac{1}{F} \bar{R}_{\nu j \nu}^{i}\right)
\end{aligned}
$$

By Lemma 2.6, we have

$$
\dot{F} \leq-\frac{F}{n}+F_{i}^{j}\left(\frac{\nabla^{i} \nabla_{j} F}{F^{2}}-2 \frac{\nabla^{i} F \nabla_{j} F}{F^{3}}-\frac{1}{F} \bar{R}_{\nu j \nu}^{i}\right)
$$
have

By Lemma 2.4, we know that $\bar{R}_{\nu j \nu}^{i}$ is uniformly bounded, together with Lemma 2.7, we

$$
-F_{i}^{j} \bar{R}_{\nu j \nu}^{i} \leq C \sum_{i} F^{i i} \leq C
$$

thus we get

$$
\dot{F}_{\max }^{2} \leq-\frac{2}{n} F_{\max }^{2}+C
$$

which gives

$$
F_{\max }^{2} \leq C
$$

Lemma 4.2. Along the flow, $F \geq c$, where $c$ depends on $\Sigma_{0}, n, k$.

Proof. Consider the function $-\log F-\log \tilde{u}$, where $\tilde{u}=u e^{-t / n}$, by Lemma2.8, $\tilde{u}$ is uniformly bounded. At the maximum point, we have

$$
\begin{aligned}
& -\frac{F_{i}}{F}-\frac{u_{i}}{u}=0,-\frac{F_{i j}}{F}+\frac{F_{i} F_{j}}{F^{2}}-\frac{u_{i j}}{u}+\frac{u_{i} u_{j}}{u^{2}} \leq 0 \\
& -\frac{F_{i}^{j}}{F} \dot{h}_{j}^{i}-\frac{\dot{u}}{u}+\frac{1}{n} \geq 0
\end{aligned}
$$

by (3.2), (3.4) and the critical equation, we have

$$
\begin{aligned}
0 & \leq-\frac{F_{i}^{j}}{F}\left(-\frac{1}{F} h_{k}^{i} h_{j}^{k}-\nabla^{i} \nabla_{j}\left(\frac{1}{F}\right)-\frac{1}{F} \bar{R}_{\nu j \nu}^{i}\right)-\frac{\phi^{\prime}}{F u}-\frac{\phi g^{i j} F_{i} r_{j}}{F^{2} u}+\frac{1}{n} \\
& =\frac{F_{i}^{j}}{F^{2}}\left(h_{k}^{i} h_{j}^{k}+\bar{R}_{\nu j \nu}^{i}\right)+\frac{g^{k i} F_{i}^{j}}{F^{2}}\left(-\frac{F_{k j}}{F}+2 \frac{F_{k} F_{j}}{F^{2}}\right)-\frac{\phi^{\prime}}{F u}-\frac{\phi g^{i j} F_{i} r_{j}}{F^{2} u}+\frac{1}{n} \\
& \leq \frac{F_{i}^{j}}{F^{2}}\left(h_{k}^{i} h_{j}^{k}+\bar{R}_{\nu j \nu}^{i}\right)+\frac{g^{k i} F_{i}^{j}}{F^{2}} \frac{u_{k j}}{u}-\frac{\phi^{\prime}}{F u}-\frac{\phi g^{i j} F_{i} r_{j}}{F^{2} u}+\frac{1}{n}
\end{aligned}
$$


by lemma 2.3, we have

$$
\begin{aligned}
0 & \leq \frac{F_{i}^{j}}{F^{2}}\left(h_{k}^{i} h_{j}^{k}+\bar{R}_{\nu j \nu}^{i}\right)+\frac{g^{k i} F_{i}^{j}}{F^{2} u}\left(g^{m n} h_{k j m} \phi r_{n}+\phi^{\prime} h_{k j}-\left(h^{2}\right)_{k j} u+g^{m n} \nabla_{m} \Phi \bar{R}_{\nu j n k}\right) \\
& -\frac{\phi^{\prime}}{F u}-\frac{\phi g^{i j} F_{i} r_{j}}{F^{2} u}+\frac{1}{n} \\
& =\frac{F_{i}^{j} \bar{R}_{\nu j \nu}^{i}}{F^{2}}+\frac{g^{k i} F_{i}^{j}}{F^{2} u} g^{m n} \nabla_{m} \Phi \bar{R}_{\nu j n k}+\frac{1}{n}
\end{aligned}
$$

by (3.5) and (3.6), we have

$$
\begin{aligned}
0 & \leq \frac{g^{k i} F_{i}^{j}}{F^{2}}\left(\left(\frac{1}{v^{2}} \delta_{k j}+\frac{2 r_{k} r_{j}}{v^{2} \phi^{2}}+\frac{r_{k} r_{j}|\nabla r|^{2}}{v^{2} \phi^{4}}\right)\left(-\phi \phi^{\prime \prime}\right)+\frac{\left(|\nabla r|^{2} \delta_{k j}-r_{k} r_{j}\right)}{v^{2} \phi^{2}}\left(1-\phi^{\prime 2}\right)\right. \\
& +\left(\frac{|\nabla r|^{2} \delta_{j k}-r_{j} r_{k}}{v^{2} \phi^{2}}\right)\left(-\phi \phi^{\prime \prime}-\left(1-\phi^{\prime 2}\right)\right)+\frac{1}{n} \\
& =\frac{g^{k i} F_{i}^{j}}{F^{2}}\left(\delta_{k j}+\frac{r_{k} r_{j}}{\phi^{2}}\right)\left(-\phi \phi^{\prime \prime}\right)+\frac{1}{n} \\
& \leq-\frac{g^{i j} F_{i}^{j}}{F^{2}} \phi \phi^{\prime \prime}+\frac{1}{n} \leq-\frac{C}{F}+\frac{1}{n}
\end{aligned}
$$

we have used the Lemma 2.7 in last line. Now we conclude that $F$ is bounded below.

Remark 4.3. For the lower bound, we only need the first inequality of Lemma 2.7, which is satisfied by a class of concave functions with homogeneous degree one, for example $F=\sigma_{k}^{1 / k}$, etc.

\section{BOUND FOR PRINCIPAL CURVATURE}

Lemma 5.1. Along the flow, $\left|\kappa_{i}\right| \leq C$ if $F$ is a hessian quotient function, where $\kappa_{i}$ is the principal curvature of $\Sigma_{t}, C$ depends on $\Sigma_{0}, n, k$.

Proof. Define $\tilde{u}=u e^{-t / n}$, consider the test function $\log (\eta)-\log (\tilde{u})$, where

$$
\eta=\sup \left\{h_{i j} \xi^{i} \xi^{j}: g_{i j} \xi^{i} \xi^{j}=1\right\}
$$

WLOG, we suppose that at the maximum point $\eta=h_{1}^{1}$, and we have

$$
\frac{\dot{h_{1}^{1}}}{h_{1}^{1}}-\frac{\dot{u}}{u}+\frac{1}{n} \geq 0
$$

and

$$
\frac{h_{1 i}^{1}}{h_{1}^{1}}-\frac{u_{i}}{u}=0, \quad \frac{h_{1 i j}^{1}}{h_{1}^{1}} \leq \frac{u_{i j}}{u}
$$


by (3.3), (3.4) and the critical equation, we have

$$
\begin{aligned}
0 & \leq \frac{1}{h_{1}^{1}}\left(-\frac{1}{F} h_{k}^{1} h_{1}^{k}+\frac{F^{p q, r s} h_{p q}{ }^{1} h_{r s 1}}{F^{2}}-\frac{2 F^{p q} h_{p q}{ }^{1} F^{r s} h_{r s 1}}{F^{3}}-\frac{1}{F} \bar{R}_{\nu 1 \nu}^{1}\right. \\
& +\frac{g^{k 1} F^{p q}}{F^{2}}\left(h_{k 1, p q}-h_{q}^{m}\left(h_{k m} h_{p 1}-h_{k 1} h_{m p}\right)-h_{1}^{m}\left(h_{m k} h_{p q}-h_{k q} h_{m p}\right)\right. \\
& \left.\left.+h_{q}^{m} \bar{R}_{k p 1 m}+h_{1}^{m} \bar{R}_{k p q m}+\nabla_{p} \bar{R}_{k 1 q \nu}+\nabla_{k} \bar{R}_{1 p q \nu}\right)\right) \\
& -\frac{\phi^{\prime}}{F u}-\frac{\phi g^{i j} F_{i} r_{j}}{F^{2} u}+\frac{1}{n}
\end{aligned}
$$

consider the term $\frac{F^{p q}}{F^{2}} \frac{h_{1, p q}^{1}}{h_{1}^{1}}$, by (5.2) and lemma 2.3, we have

$$
\frac{F^{p q}}{F^{2}} \frac{h_{1, p q}^{1}}{h_{1}^{1}} \leq \frac{F^{p q}}{F^{2}} \frac{u_{p q}}{u}=\frac{F^{p q}}{F^{2} u}\left(g^{k l} h_{p q k} \Phi_{l}+\phi^{\prime} h_{p q}-\left(h^{2}\right)_{p q} u+g^{k l} \nabla_{l} \Phi \bar{R}_{\nu p k q}\right)
$$

insert (5.4) into (5.3), together with the concavity of $F$, yields

$$
\begin{aligned}
0 & \leq \frac{1}{h_{1}^{1}}\left(-\frac{1}{F} h_{k}^{1} h_{1}^{k}-\frac{1}{F} \bar{R}_{\nu 1 \nu}^{1}+\frac{g^{k 1} F^{p q}}{F^{2}}\left(-h_{1}^{m} h_{m k} h_{p q}+h_{q}^{m} \bar{R}_{k p 1 m}+h_{1}^{m} \bar{R}_{k p q m}+\nabla_{p} \bar{R}_{k 1 q \nu}+\nabla_{k} \bar{R}_{1 p q \nu}\right)\right) \\
& +\frac{g^{k l} F^{p q}}{F^{2} u} \nabla_{l} \Phi \bar{R}_{\nu p k q}+\frac{1}{n}
\end{aligned}
$$

Using the fact $1-\phi^{\prime 2}+\phi \phi^{\prime \prime} \geq 0$, together with (3.6)

$$
g^{k l} \nabla_{l} \Phi \bar{R}_{\nu p k q}=\left(\frac{|\nabla r|^{2} \delta_{p q}-r_{p} r_{q}}{v^{3} \phi}\right)\left(-\phi \phi^{\prime \prime}-\left(1-\phi^{\prime 2}\right)\right) \leq 0
$$

thus we have

$$
0 \leq \frac{1}{h_{1}^{1}}\left(-\frac{2}{F} h_{k}^{1} h_{1}^{k}-\frac{1}{F} \bar{R}_{\nu 1 \nu}^{1}+\frac{g^{k 1} F^{p q}}{F^{2}}\left(h_{q}^{m} \bar{R}_{k p 1 m}+h_{1}^{m} \bar{R}_{k p q m}+\nabla_{p} \bar{R}_{k 1 q \nu}+\nabla_{k} \bar{R}_{1 p q \nu}\right)\right)+\frac{1}{n}
$$

By Lemma 2.5. all terms involving curvature terms of the ambient space are uniformly bounded, i.e.

$$
h_{q}^{m} \bar{R}_{k p 1 m}+h_{1}^{m} \bar{R}_{k p q m}+\nabla_{p} \bar{R}_{k 1 q \nu}+\nabla_{k} \bar{R}_{1 p q \nu} \leq C h_{1}^{1}+C
$$

By Lemma 2.7 and the lower bound of $F$ Lemma 4.2,

$$
\frac{g^{k 1} F^{p q}}{F^{2}}\left(h_{q}^{m} \bar{R}_{k p 1 m}+h_{1}^{m} \bar{R}_{k p q m}+\nabla_{p} \bar{R}_{k 1 q \nu}+\nabla_{k} \bar{R}_{1 p q \nu}\right) \leq C h_{1}^{1}+C
$$

Plug into (5.7), together with the upper bound of $F$ Lemma 4.1 yields

$$
0 \leq-C h_{1}^{1}+C
$$

i.e. $h_{1}^{1} \leq C$, thus we have the lemma.

Corollary 5.2. The solution of the inverse curvature flow exists for all time. 
Proof. We have established up to $C^{2}$ apriori estimate, by Lemma 5.1, $F$ is uniformly elliptic, by Evans-Krylov theorem, we have $C^{2, \alpha}$ estimate, together with Schauder estimate, we have all the high order estimates, the corollary now follows.

\section{Asmptotic Behavior of SECOND FUndamental FORM}

In this section, we consider the asmptotic behaviour of second fundamental form, the test function was first considered by Scheuer in [18.

\section{Lemma 6.1.}

$$
\limsup _{t \rightarrow \infty} \sup _{i} \kappa_{i} \leq 1
$$

where $\kappa_{i}$ is the principal curvature of $M$.

Proof. Let's consider the test function $w=(\log \eta-\log \tilde{u}+r-\log 2) t$, where

$$
\eta=\sup \left\{h_{i j} \xi^{i} \xi^{j}: g_{i j} \xi^{i} \xi^{j}=1\right\}
$$

Noting that

$$
(-\log \tilde{u}+r-\log 2) t=(\log v-\log \phi+r-\log 2) t
$$

by Lemma 3.2, $t \log v \leq C$. By Lemma 2.5, we have

$$
\phi \geq \frac{e^{r}}{2}-C e^{-r}
$$

thus

$$
(-\log \phi+r-\log 2) t \leq t \log \frac{e^{r}}{e^{r}-C e^{-r}} \leq t \log \left(1+C e^{-2 r}\right) \leq C
$$

i.e.

$$
(-\log \tilde{u}+r-\log 2) t \leq C
$$

Similarly,

$$
(-\log \tilde{u}+r-\log 2) t \geq-C
$$

WLOG, we suppose that at the maximum point of $w$, say $\left(x_{0}, t_{0}\right), \eta=h_{1}^{1}$, and we have

$$
0 \leq\left(\frac{\dot{h_{1}^{1}}}{h_{1}^{1}}-\frac{\dot{u}}{u}+\dot{r}\right) t+\left(\log h_{1}^{1}-\log \tilde{u}+r-\log 2\right)
$$

and

$$
\begin{aligned}
& \frac{h_{1 i}^{1}}{h_{1}^{1}}-\frac{u_{i}}{u}+r_{i}=0 \\
& \frac{h_{1 i j}^{1}}{h_{1}^{1}}-\frac{h_{1 i}^{1} h_{1 j}^{1}}{\left(h_{1}^{1}\right)^{2}}-\frac{u_{i j}}{u}+\frac{u_{i} u_{j}}{u^{2}}+r_{i j} \leq 0
\end{aligned}
$$


INVERSE CURVATURE FLOW IN ANTI-DE SITTER-SCHWARZSCHILD MANIFOLD

by (2.9), (3.3), (3.4) and the critical equation, we have

$$
\begin{aligned}
0 & \leq \frac{t_{0}}{h_{1}^{1}}\left(-\frac{1}{F} h_{k}^{1} h_{1}^{k}+\frac{F^{p q, r s} h_{p q}{ }^{1} h_{r s 1}}{F^{2}}-\frac{2 F^{p q} h_{p q}{ }^{1} F^{r s} h_{r s 1}}{F^{3}}-\frac{1}{F} \bar{R}_{\nu 1 \nu}^{1}\right. \\
& +\frac{g^{k 1} F^{p q}}{F^{2}}\left(h_{k 1, p q}-h_{q}^{m}\left(h_{k m} h_{p 1}-h_{k 1} h_{m p}\right)-h_{1}^{m}\left(h_{m k} h_{p q}-h_{k q} h_{m p}\right)\right. \\
& \left.\left.+h_{q}^{m} \bar{R}_{k p 1 m}+h_{1}^{m} \bar{R}_{k p q m}+\nabla_{p} \bar{R}_{k 1 q \nu}+\nabla_{k} \bar{R}_{1 p q \nu}\right)\right) \\
& -\frac{t_{0}}{u}\left(\frac{\phi^{\prime}}{F}+\frac{\phi g^{i j} F_{i} r_{j}}{F^{2}}\right)+\frac{v t_{0}}{F}+\left(\log h_{1}^{1}-\log \tilde{u}+\tilde{r}-\log 2\right)
\end{aligned}
$$

i.e.

$$
\begin{aligned}
0 & \leq \frac{t_{0}}{h_{1}^{1}}\left(-\frac{2}{F} h_{k}^{1} h_{1}^{k}-\frac{1}{F} \bar{R}_{\nu 1 \nu}^{1}+\frac{g^{k 1} F^{p q}}{F^{2}}\left(h_{k 1, p q}+h_{q}^{m} h_{k 1} h_{m p}\right.\right. \\
& \left.\left.+h_{q}^{m} \bar{R}_{k p 1 m}+h_{1}^{m} \bar{R}_{k p q m}+\nabla_{p} \bar{R}_{k 1 q \nu}+\nabla_{k} \bar{R}_{1 p q \nu}\right)\right) \\
& -\frac{t_{0}}{u}\left(\frac{\phi^{\prime}}{F}+\frac{\phi g^{i j} F_{i} r_{j}}{F^{2}}\right)+\frac{v t_{0}}{F}+C
\end{aligned}
$$

consider the term $\frac{F^{p q}}{F^{2}} \frac{h_{1, p q}^{1}}{h_{1}^{1}}$, by (2.10), Lemma 2.3 and the critical equation we have

$$
\begin{aligned}
\frac{F^{p q}}{F^{2}} \frac{h_{1, p q}^{1}}{h_{1}^{1}} & \leq \frac{F^{p q}}{F^{2}}\left(\frac{u_{p q}}{u}+\frac{h_{1 p}^{1} h_{1 q}^{1}}{\left(h_{1}^{1}\right)^{2}}-\frac{u_{p} u_{q}}{u^{2}}-r_{p q}\right) \\
& =\frac{F^{p q}}{F^{2} u}\left(g^{k l} h_{p q k} \Phi_{l}+\phi^{\prime} h_{p q}-\left(h^{2}\right)_{p q} u+g^{k l} \nabla_{l} \Phi \bar{R}_{\nu p k q}\right) \\
& +\frac{F^{p q}}{F^{2}}\left(h_{p q} v-\phi \phi^{\prime} \delta_{p q}-\frac{2 \phi^{\prime} r_{p} r_{q}}{\phi}\right)+\frac{F^{p q}}{F^{2}}\left(\frac{h_{1 p}^{1} h_{1 q}^{1}}{\left(h_{1}^{1}\right)^{2}}-\frac{u_{p} u_{q}}{u^{2}}\right)
\end{aligned}
$$

plug into (6.5), we have

$$
\begin{aligned}
0 & \leq \frac{t_{0}}{h_{1}^{1}}\left(-\frac{2}{F} h_{k}^{1} h_{1}^{k}-\frac{1}{F} \bar{R}_{\nu 1 \nu}^{1}+\frac{g^{k 1} F^{p q}}{F^{2}}\left(h_{q}^{m} \bar{R}_{k p 1 m}+h_{1}^{m} \bar{R}_{k p q m}+\nabla_{p} \bar{R}_{k 1 q \nu}+\nabla_{k} \bar{R}_{1 p q \nu}\right)\right) \\
& +\frac{t_{0} g^{k l} F^{p q}}{F^{2} u} \nabla_{l} \Phi \bar{R}_{\nu p k q}-\frac{t_{0} F^{p q}}{F^{2}}\left(\phi \phi^{\prime} \delta_{p q}+\frac{2 \phi^{\prime} r_{p} r_{q}}{\phi}\right)+\frac{t_{0} F^{p q}}{F^{2}}\left(\frac{h_{1 p}^{1} h_{1 q}^{1}}{\left(h_{1}^{1}\right)^{2}}-\frac{u_{p} u_{q}}{u^{2}}\right)+\frac{2 v t_{0}}{F}+C
\end{aligned}
$$

by Lemma 2.5 and Lemma 3.2, we have

$$
\begin{aligned}
& \frac{g^{k 1} F^{p q}}{F^{2}}\left(h_{q}^{m} \bar{R}_{k p 1 m}+h_{1}^{m} \bar{R}_{k p q m}+\nabla_{p} \bar{R}_{k 1 q \nu}+\nabla_{k} \bar{R}_{1 p q \nu}\right) \\
= & \frac{F_{p}^{p}}{F^{2}}\left(-h_{p}^{p}+h_{1}^{1}\right)+O\left(e^{-\alpha t_{0}}\right) \\
= & -\frac{1}{F}+\frac{F_{p}^{p}}{F^{2}} h_{1}^{1}+O\left(e^{-\alpha t_{0}}\right)
\end{aligned}
$$


similarly,

$$
-\frac{1}{F} \bar{R}_{\nu 1 \nu}^{1}=\frac{1}{F}+O\left(e^{-\alpha t_{0}}\right), \quad \frac{g^{k l} F^{p q}}{F^{2} u} \nabla_{l} \Phi \bar{R}_{\nu p k q}=O\left(e^{-\alpha t_{0}}\right)
$$

Plug into (6.7), we have

$$
\begin{aligned}
0 & \leq \frac{t_{0}}{h_{1}^{1}}\left(-\frac{2}{F} h_{k}^{1} h_{1}^{k}+\frac{F_{p}^{p}}{F^{2}} h_{1}^{1}\right)+\frac{2 v t_{0}}{F}+C \\
& -\frac{t_{0} F^{p q}}{F^{2}}\left(\phi \phi^{\prime} \delta_{p q}+\frac{2 \phi^{\prime} r_{p} r_{q}}{\phi}\right)+\frac{t_{0} F^{p q}}{F^{2}}\left(\frac{h_{1 p}^{1} h_{1 q}^{1}}{\left(h_{1}^{1}\right)^{2}}-\frac{u_{p} u_{q}}{u^{2}}\right)
\end{aligned}
$$

By the critical equation, we have

$$
\frac{F^{p q}}{F^{2}}\left(\frac{h_{1 p}^{1} h_{1 q}^{1}}{\left(h_{1}^{1}\right)^{2}}-\frac{u_{p} u_{q}}{u^{2}}\right)=\frac{F^{p q}}{F^{2}}\left(-\frac{2 u_{p} r_{q}}{u}+r_{p} r_{q}\right)
$$

Since

$$
\nabla_{i} u=g^{k l} h_{i k} \nabla_{l} \Phi=g^{k l} h_{i k} \phi r_{l}
$$

together with Lemma 3.2, we have

$$
\frac{t_{0} F^{p q}}{F^{2}}\left(\frac{h_{1 p}^{1} h_{1 q}^{1}}{\left(h_{1}^{1}\right)^{2}}-\frac{u_{p} u_{q}}{u^{2}}\right) \leq C
$$

thus

$$
\begin{aligned}
0 & \leq \frac{t_{0}}{h_{1}^{1}}\left(-\frac{2}{F} h_{k}^{1} h_{1}^{k}+\frac{F_{p}^{p}}{F^{2}} h_{1}^{1}\right)+\frac{2 v t_{0}}{F}+C \\
& -\frac{t_{0} F^{p q}}{F^{2}}\left(\phi \phi^{\prime} \delta_{p q}+\frac{2 \phi^{\prime} r_{p} r_{q}}{\phi}\right)
\end{aligned}
$$

Again by Lemma 3.2 and the relation $\phi^{\prime}=\phi+O(1)$, we have

$$
\begin{aligned}
0 & \leq \frac{t_{0}}{h_{1}^{1}}\left(-\frac{2}{F} h_{k}^{1} h_{1}^{k}+\frac{F_{p}^{p}}{F^{2}} h_{1}^{1}\right)+\frac{2 t_{0}}{F}+C-\frac{t_{0} F_{p}^{p}}{F^{2}} \\
& =-\frac{2 t_{0}}{F} h_{1}^{1}+\frac{2 t_{0}}{F}+C
\end{aligned}
$$

thus

$$
h_{1}^{1}-1 \leq \frac{C}{t_{0}}
$$

we have

$$
w \leq t_{0} \log \left(1+\frac{C}{t_{0}}\right)+t_{0}(-\log \tilde{u}+\tilde{r}-\log 2) \leq C
$$

thus

$$
\left(\log h_{1}^{1}-\log \tilde{u}+\tilde{r}-\log 2\right) t \leq C
$$

for any $t$, together with (6.2), we have

$$
\limsup _{t \rightarrow \infty} \sup _{M} \kappa_{i}(t, \cdot) \leq 1
$$


Lemma 6.2. $F \geq n-C t e^{-2 \alpha t}$, where $C$ depends on $\Sigma_{0}, n, k$.

Proof. Consider the test function $w=\frac{v}{F}$, thus $\dot{\varphi}=\frac{1}{G}=\frac{w}{\phi}$, we have

$$
\frac{\partial w}{\partial t}=\phi \frac{\partial \dot{\varphi}}{\partial t}+\phi \phi^{\prime} \dot{\varphi}^{2}
$$

Let $G^{i j}=\frac{\partial G}{\partial \varphi_{i j}}, G^{k}=\frac{\partial G}{\partial \varphi_{k}}$, then

$$
G^{i j}=-\frac{1}{v^{2}} F_{l}^{i} \tilde{\sigma}^{l j}
$$

similar to Lemma 3.1, we have

$$
\begin{aligned}
\frac{\partial w}{\partial t} & =\frac{\phi}{v^{2} G^{2}}\left(F_{l}^{i} \tilde{\sigma}^{l j} \dot{\varphi}_{i j}-v^{2} G^{k} \dot{\varphi}_{k}-F_{i}^{i} \phi \phi^{\prime \prime} \dot{\varphi}\right)+\frac{\phi^{\prime}}{\phi} w^{2} \\
& =\frac{w^{2}}{v^{2} \phi}\left(F_{l}^{i} \tilde{\sigma}^{l j}\left(\frac{w}{\phi}\right)_{i j}-v^{2} G^{k}\left(\frac{w}{\phi}\right)_{k}-F_{i}^{i} \phi^{\prime \prime} w\right)+\frac{\phi^{\prime}}{\phi} w^{2} \\
& =\frac{w^{2}}{v^{2} \phi^{2}}\left(F_{l}^{i} \tilde{\sigma}^{l j} w_{i j}-\frac{2}{\phi} F_{l}^{i} \tilde{\sigma}^{l j} w_{i} \phi_{j}-v^{2} G^{k} w_{k}\right) \\
& +\frac{w^{2}}{v^{2} \phi^{2}}\left(\frac{2 w}{\phi^{2}} F_{l}^{i} \tilde{\sigma}^{l j} \phi_{i} \phi_{j}-\frac{w}{\phi} F_{l}^{i} \tilde{\sigma}^{l j} \phi_{i j}+\frac{v^{2} w}{\phi} G^{k} \phi_{k}\right) \\
& +\frac{\phi^{\prime}}{\phi} w^{2}-\frac{F_{i}^{i} \phi^{\prime \prime}}{v^{2} \phi} w^{3}
\end{aligned}
$$

First, note that $w$ is bounded by our previous estimate, thus we only need to consider the second line.

By Lemma 3.2, We have

$$
\frac{2 w}{\phi^{2}} F_{l}^{i} \tilde{\sigma}^{l j} \phi_{i} \phi_{j} \leq C e^{\left(\frac{2}{n}-\alpha\right) t}
$$

Now by (2.12)

$$
\begin{aligned}
\phi_{i j} & =\phi^{\prime} r_{i j}+\phi^{\prime \prime} r_{i} r_{j} \\
& =\phi \phi^{\prime} \varphi_{i j}+\phi\left(\phi^{\prime 2}+\phi \phi^{\prime \prime}\right) \varphi_{i} \varphi_{j}
\end{aligned}
$$

thus

$$
\begin{aligned}
-F_{l}^{i} \tilde{\sigma}^{l j} \phi_{i j} & =-\phi \phi^{\prime} F_{l}^{i} \tilde{\sigma}^{l j} \varphi_{i j}-\phi\left({\phi^{\prime}}^{2}+\phi \phi^{\prime \prime}\right) F_{l}^{i} \tilde{\sigma}^{l j} \varphi_{i} \varphi_{j} \\
& \leq-\phi \phi^{\prime} F_{l}^{i}\left(\phi^{\prime} \delta_{i}^{l}-\phi v h_{i}^{l}\right)+C e^{\left(\frac{3}{n}-2 \alpha\right) t}
\end{aligned}
$$

By lemma 2.7.

$$
\begin{aligned}
-F_{l}^{i} \tilde{\sigma}^{l j} \phi_{i j} & \leq-\phi \phi^{\prime}\left(n \phi^{\prime}-\phi v F\right)+C e^{\left(\frac{3}{n}-2 \alpha\right) t} \\
& =\phi \phi^{\prime}\left(v^{2} \frac{\phi}{w}-n \phi^{\prime}\right)+C e^{\left(\frac{3}{n}-2 \alpha\right) t}
\end{aligned}
$$

i.e.

$$
-\frac{w}{\phi} F_{l}^{i} \tilde{\sigma}^{l j} \phi_{i j} \leq \phi \phi^{\prime} v^{2}-n \phi^{\prime 2} w+C e^{\left(\frac{2}{n}-2 \alpha\right) t}
$$


Now, consider $G^{k}$, we have

$$
G^{k}=\frac{F_{k}^{i} \varphi_{i j}}{v^{2}} \frac{\varphi^{i}}{v^{2}}-2 \frac{F_{l}^{i} \varphi_{i j}}{v^{2}} \frac{\varphi^{l} \varphi^{j} \varphi^{k}}{v^{4}}-2 \frac{F}{v^{4}} \varphi^{k}
$$

since $h_{j}^{i}$ is bounded, by (2.15), we have $\left|\varphi_{i j}\right| \leq C e^{\frac{t}{n}}$. thus

$$
G^{k} \phi_{k}=\phi \phi^{\prime} G^{k} \varphi_{k}=\phi \phi^{\prime}\left(\frac{F_{k}^{i} \varphi_{i j}}{v^{4}} \varphi^{j} \varphi_{k}-2 \frac{F_{l}^{i} \varphi_{i j}}{v^{6}} \varphi^{l} \varphi^{j}|\nabla \varphi|^{2}-2 \frac{F}{v^{4}}|\nabla \varphi|^{2}\right) \leq C e^{\left(\frac{3}{n}-2 \alpha\right) t}
$$

Put all together, we have

$$
\begin{aligned}
\frac{\partial w}{\partial t} & \leq \frac{w^{2}}{v^{2} \phi^{2}}\left(F_{l}^{i} \tilde{\sigma}^{l j} w_{i j}-\frac{2}{\phi} F_{l}^{i} \tilde{\sigma}^{l j} w_{i} \phi_{j}-v^{2} G^{k} w_{k}\right) \\
& +\frac{w^{2}}{v^{2} \phi^{2}}\left(\phi \phi^{\prime} v^{2}-n \phi^{\prime 2} w\right)+\frac{\phi^{\prime}}{\phi} w^{2}-\frac{F_{i}^{i} \phi^{\prime \prime}}{v^{2} \phi} w^{3}+C e^{-2 \alpha t} \\
& \leq \frac{w^{2}}{v^{2} \phi^{2}}\left(F_{l}^{i} \tilde{\sigma}^{l j} w_{i j}-\frac{2}{\phi} F_{l}^{i} \tilde{\sigma}^{l j} w_{i} \phi_{j}-v^{2} G^{k} w_{k}\right) \\
& +2 \frac{\phi^{\prime}}{\phi} w^{2}-n \frac{\phi \phi^{\prime \prime}+\phi^{\prime 2}}{v^{2} \phi^{2}} w^{3}+C e^{-2 \alpha t}
\end{aligned}
$$

by Lemma 3.2 and Lemma 2.5 , we have

$$
\frac{d}{d t} w_{\max } \leq 2 w_{\max }^{2}-2 n w_{\max }^{3}+C e^{-2 \alpha t}
$$

By Lemma 6.1, we have $w_{\max } \geq \frac{1}{n}$, thus

$$
\frac{d}{d t} w_{\max } \leq \frac{2}{n^{2}}-\frac{2}{n} w_{\max }+C e^{-2 \alpha t}
$$

thus

$$
w_{\max } \leq \frac{1}{n}+C t e^{-2 \alpha t}
$$

thus

$$
F \geq n-C t e^{-2 \alpha t}
$$

Put lemma 6.1 and lemma 6.2 together, we have

\section{Corollary 6.3.}

$$
\left|h_{j}^{i}-\delta_{j}^{i}\right| \rightarrow 0
$$

as $t \rightarrow \infty$

Now let's compute the convergence rate, we have the following lemma,

\section{Lemma 6.4.}

$$
\left|h_{j}^{i}-\delta_{j}^{i}\right| \leq O\left(e^{-\frac{2}{n} t}\right) .
$$


Proof. Consider the test function

$$
G=\frac{1}{2} \sum_{i j}\left(h_{j}^{i}-\delta_{j}^{i}\right)\left(h_{i}^{j}-\delta_{i}^{j}\right) e^{\lambda t}
$$

we have

$$
\dot{G}=\sum_{i j} \dot{h}_{j}^{i}\left(h_{i}^{j}-\delta_{i}^{j}\right) e^{\lambda t}+\lambda G
$$

for each $t, G$ attains maximum at some point $x_{0}$, at $x_{0}$

$$
\begin{aligned}
& \sum_{i j} h_{j k}^{i}\left(h_{i}^{j}-\delta_{i}^{j}\right)=0 \\
& \sum_{i j} h_{j k l}^{i}\left(h_{i}^{j}-\delta_{i}^{j}\right)+h_{j k}^{i} h_{i l}^{j} \leq 0
\end{aligned}
$$

thus

$$
\begin{aligned}
\dot{G} & =\left(-\frac{1}{F} h_{k}^{i} h_{j}^{k}+\frac{F^{p q, r s} h_{p q}{ }^{i} h_{r s j}}{F^{2}}-\frac{2 F^{p q} h_{p q}{ }^{i} F^{r s} h_{r s j}}{F^{3}}-\frac{1}{F} \bar{R}_{\nu j \nu}^{i}\right. \\
& +\frac{g^{k i} F^{p q}}{F^{2}}\left(h_{k j, p q}-h_{q}^{m}\left(h_{k m} h_{p j}-h_{k j} h_{m p}\right)-h_{j}^{m}\left(h_{m k} h_{p q}-h_{k q} h_{m p}\right)\right. \\
& \left.\left.+h_{q}^{m} \bar{R}_{k p j m}+h_{j}^{m} \bar{R}_{k p q m}+\nabla_{p} \bar{R}_{k j q \nu}+\nabla_{k} \bar{R}_{j p q \nu}\right)\right)\left(h_{i}^{j}-\delta_{i}^{j}\right) e^{\lambda t}+\lambda G
\end{aligned}
$$

by the critical equation, we have

$$
\begin{aligned}
\dot{G} & \leq\left(-\frac{1}{F} h_{k}^{i} h_{j}^{k}+\frac{F^{p q, r s} h_{p q}{ }^{i} h_{r s j}}{F^{2}}-\frac{2 F^{p q} h_{p q}{ }^{i} F^{r s} h_{r s j}}{F^{3}}-\frac{1}{F} \bar{R}_{\nu j \nu}^{i}\right. \\
& +\frac{g^{k i} F^{p q}}{F^{2}}\left(-h_{q}^{m}\left(h_{k m} h_{p j}-h_{k j} h_{m p}\right)-h_{j}^{m}\left(h_{m k} h_{p q}-h_{k q} h_{m p}\right)\right. \\
& \left.\left.+h_{q}^{m} \bar{R}_{k p j m}+h_{j}^{m} \bar{R}_{k p q m}+\nabla_{p} \bar{R}_{k j q \nu}+\nabla_{k} \bar{R}_{j p q \nu}\right)\right)\left(h_{i}^{j}-\delta_{i}^{j}\right) e^{\lambda t}-\frac{F^{p q}}{F^{2}} h_{j p}^{i} h_{i q}^{j} e^{\lambda t}+\lambda G
\end{aligned}
$$

by Corollary 6.3, all the terms involving the derivatives of $h_{j}^{i}$ can be controlled by $-\frac{F^{p q}}{F^{2}} h_{j p}^{i} h_{i q}^{j}$, thus

$$
\begin{aligned}
\dot{G} & \leq\left(-\frac{1}{F} h_{k}^{i} h_{j}^{k}-\frac{1}{F} \bar{R}_{\nu j \nu}^{i}+\frac{g^{k i} F^{p q}}{F^{2}}\left(-h_{q}^{m}\left(h_{k m} h_{p j}-h_{k j} h_{m p}\right)-h_{j}^{m}\left(h_{m k} h_{p q}-h_{k q} h_{m p}\right)\right.\right. \\
& \left.\left.+h_{q}^{m} \bar{R}_{k p j m}+h_{j}^{m} \bar{R}_{k p q m}+\nabla_{p} \bar{R}_{k j q \nu}+\nabla_{k} \bar{R}_{j p q \nu}\right)\right)\left(h_{i}^{j}-\delta_{i}^{j}\right) e^{\lambda t}+\lambda G
\end{aligned}
$$

Diagonalized it, we have

$$
g_{i j}=\delta_{i j}, h_{i j}=\kappa_{i} \delta_{i j}, \kappa_{1} \leq \cdots \leq \kappa_{n}
$$


and by Lemma 2.5, Lemma 3.2 and Lemma 6.1, we have

$$
\begin{aligned}
\dot{G} & \leq\left(-\frac{1}{F} \kappa_{i}^{2}+\frac{F^{p p}}{F^{2}}\left(\kappa_{i} \kappa_{p}^{2}-\kappa_{i}^{2} \kappa_{p}+\kappa_{i}\right)+c e^{-\frac{2}{n} t}\right)\left(\kappa_{i}-1\right) e^{\lambda t}+\lambda G \\
& =\left(-\frac{2}{F}\left(\kappa_{i}^{2}-\kappa_{i}\right)+\frac{F^{p p}}{F^{2}} \kappa_{i}\left(\kappa_{p}-1\right)^{2}+c e^{-\frac{2}{n} t}\right)\left(\kappa_{i}-1\right) e^{\lambda t}+\lambda G \\
& \leq\left(-\frac{4}{F} \kappa_{i}+\lambda+2 \frac{F^{p p}}{F^{2}} \kappa_{i}\left|\kappa_{i}-1\right|\right) G+c\left(\kappa_{i}-1\right) e^{\left(-\frac{2}{n}+\lambda\right) t}
\end{aligned}
$$

Thus if we choose $\lambda$ small enough, we conclude that $G$ is bounded, i.e. $\left|h_{j}^{i}-\delta_{j}^{i}\right|=O\left(e^{-\frac{\lambda}{2} t}\right)$ for small $\lambda$.

Now if we choose $\tilde{G}=\sup _{M} \frac{1}{2}\left|h_{j}^{i}-\delta_{j}^{i}\right|^{2} e^{\frac{4 t}{n}}$, we have

$$
\begin{aligned}
\dot{\tilde{G}} & \leq\left(-\frac{4}{F} \kappa_{i}+\frac{4}{n}+2 \frac{F^{p p}}{F^{2}} \kappa_{i}\left|\kappa_{i}-1\right|\right) \tilde{G}+c e^{-\frac{\lambda t}{2}} \\
& \leq c e^{-\frac{\lambda t}{2}} \tilde{G}+c e^{-\frac{\lambda t}{2}}
\end{aligned}
$$

write $\sqrt{\tilde{G}}=f$, we have

$$
\dot{f} \leq c e^{-\frac{\lambda t}{2}} f+c e^{-\frac{\lambda t}{2}}
$$

thus $f \leq C$, we proved the lemma.

Acknowledgement: The author would like to express gratitude to his supervisor Professor Pengfei Guan for consistent support and encouragement.

\section{REFERENCES}

[1] B. Andrews, Contraction of convex hypersurfaces in Euclidean space, Calc. Var. Partial Differential Equations 2 (1994), no. 2, 151-171.

[2] B. Andrews, Contraction of convex hypersurfaces in Riemannian spaces, J. Differential Geom. 39 (1994), no. 2, 407-431.

[3] S. Brendle, P.-K. Huang and M.-T. Wang, A Minkowski inequality for hypersurfaces in the anti-deSitterSchwarzschild manifold, Comm. Pure Appl. Math. 69 (2016), no. 1, 124-144.

[4] S. Brendle and M.-T. Wang, A Gibbons-Penrose inequality for surfaces in Schwarzschild spacetime, Comm. Math. Phys. 330 (2014), no. 1, 33-43.

[5] L. Chen and J. Mao, The long-time convergence of inverse curvature flows in the anti-de Sitter Schwarzschild manifold, arXiv:1610.00836

[6] Q. Ding, The inverse mean curvature flow in rotationally symmetric spaces, Chin. Ann. Math. 32(B), $2011,27-44$.

[7] C. Gerhardt, Flow of nonconvex hypersurfaces into spheres, J. Differential Geometry 32 (1990) 299-314.

[8] C. Gerhardt, Inverse curvature flows in hyperbolic space, J. Differential Geometry 89 (2011) 487-527.

[9] C. Gerhardt, Curvature flows in the sphere, J. Differential Geom. 100 (2015), no. 2, 301-347.

[10] P. Guan and J. Li, The quermassintegral inequalities for $k$-convex starshaped domains, Adv. Math. 221 (2009), no. 5, 1725-1732.

[11] P. Guan and J. Li, A mean curvature type flow in space forms, International Mathematics Research Notices, Vol. 2015, no. 13, (2015) 4716-4740.

[12] Y. Ge, G. Wang and J. Wu, Hyperbolic Alexandrov-Fenchel quermassintegral inequalities II, J. Differential Geom. 98 (2014), no. 2, 237-260.

[13] G. Huisken, Gerhard Flow by mean curvature of convex surfaces into spheres, J. Differential Geom. 20 (1984), no. 1, 237-266.

[14] G. Huisken, Contracting convex hypersurfaces in Riemannian manifolds by their mean curvature, Invent. Math. 84 (1986), no. 3, 463-480. 
[15] P.-K. Hung and M.-T. Wang, Inverse mean curvature flows in the hyperbolic 3-space revisited, Calc. Var. Partial Differential Equations 54 (2015), no. 1, 119-126.

[16] H. Li, Y. Wei and C. Xiong, A geometric inequality on hypersurface in hyperbolic space, Adv. Math. 253 (2014), 152-162.

[17] A. Neves, Insufficient convergence of inverse mean curvature flow on asymptotically hyperbolic manifolds, J. Differential Geom. 84 (2010), no. 1, 191-229.

[18] J. Scheuer, Non-scale-invariant inverse curvature flows in hyperbolic space, Calc. Var. Partial Differential Equations 53 (2015), no. 1-2, 91-123.

[19] J. Scheuer, The inverse mean curvature flow in warped cylinders of non-positive radial curvature, Adv. Math. 306 (2017), 1130-1163.

[20] J. Urbas, On the expansion of starshaped hypersurfaces by symmetric functions of their principal curvatures, Math. Z. 205, 355-372(1990).

Department of Mathematics and Statistics, McGill University, 805 Sherbrooke O, MonTreal, Quebec, CANAdA, H3A 0B9

E-mail address: siyuan.lu@mail.mcgill.ca 